\title{
Selective reinstatement of stimulus-outcome associations
}

\author{
ANDREW R. DELAMATER \\ Brooklyn College-CUNY, Brooklyn, New York
}

\begin{abstract}
In two experiments, the possibility of outcome-selective reinstatement of conditioned responding was examined. Evidence for outcome-selective reinstatement of previously extinguished appetitively conditioned magazine responses by rats was observed in both Pavlovian (Experiment 1) and discriminated instrumental conditioning (Experiment 2) procedures. In both experiments, stimulus-elicited magazine responses occurred more in the presence of a stimulus whose reinforcer was reinstated than they did in the presence of another stimulus whose reinforcer was not reinstated. This effect was observed after both brief and extensive amounts of extinction. Outcome-selective reinstatement of instrumental leverpressing, however, was not observed, although nonselective reinstatement of magazine responding and leverpressing was obtained in Experiment 2. Overall, the data from these studies challenge existing theories of reinstatement, and they provide additional evidence of the importance of outcome-specific processes in the control of learned performance.
\end{abstract}

The presentation of noncontingent reinforcement after a learned response has been extinguished results in a restoration of conditioned responding. This "reinstatement" of conditioned responding (Rescorla \& Heth, 1975) is a rather robust phenomenon, having been observed in both appetitive (e.g., Bouton \& Peck, 1989) and aversive (e.g., Bouton \& Bolles, 1979; Schachtman, Brown, \& Miller, 1985) conditioning situations as well as in Pavlovian and instrumental (e.g., Baker, Steinwald, \& Bouton, 1991; Rescorla \& Skucy, 1969) conditioning paradigms. Indeed, in the substance-abuse literature, the phenomenon is sometimes regarded as one of the determinants of relapse to drug taking (e.g., Shaham, Rodaros, \& Stewart, 1994; Shaham \& Stewart, 1995). Given the generality of this phenomenon, it is perhaps not surprising that a variety of theoretical explanations have been advanced to account for reinstatement. In spite of the rather extensive analyses that the phenomenon of reinstatement has received, there is no widespread agreement as to how it is best explained.

The dominant models of reinstatement emphasize the importance of context in elevating conditioned responding during the test for reinstatement. For example, the context-summation view asserts that presentations of the unconditioned stimulus (US) after a conditioning and extinction series but before the test phase strengthens asso-

This research was supported through an NSF grant (BNS-88-03514) awarded to R. A. Rescorla, and through an NRSA postdoctoral fellowship (MH-10007) awarded to the author. The helpful comments of Bob Rescorla, Peter Urcuioli, and two anonymous reviewers on previous versions of this manuscript are gratefully acknowledged, as is the assistance of Anastasia Droungas in the preparation of this manuscript. Reprint requests may be directed to the author at Brooklyn CollegeCUNY, Psychology Department, 2900 Bedford Ave., Brooklyn, NY 11210 (e-mail: andrewd@james1.brooklyn.cuny.edu). ciations between the context and the reinforcer. In a Pavlovian paradigm, when the extinguished conditioned stimulus (CS) is tested in this context, residual associative strength to the CS is assumed to summate with the increased associative strength of the context to produce an increase in conditioned responding. Although this model receives support from Bouton and Bolles's (1979) demonstration that the amount of reinstatement depends upon the amount of contextual excitation (for similar evidence in an instrumental conditioning situation, see Baker et al., 1991), Bouton and his colleagues have shown that contextual excitation does not always summate with CSs to elevate responding. In particular, Bouton and King (1986; also see Bouton, Rosengard, Achenbach, Peck, \& Brooks, 1993) demonstrated that for conditioned responding to be reinstated to a CS, that CS must have a prior history of extinction and depressed performance going into the test. An extinguished CS was more effectively reinstated when the reinstating US had been presented in the test context than when it had been presented in an alternative context. However, partially reinforced, latently inhibited, and extinguished but then substantially retrained CSs were all insensitive to the associative strength of the test context in spite of the fact that care was taken to ensure that conditioned responding was not at a ceiling prior to these tests.

These observations have prompted Bouton and his colleagues (for a review, see Bouton, 1991) to reject the context-CS summation view and to interpret reinstatement, instead, as a special form of occasion-setting by contextual cues. The idea is that contextual stimuli can acquire conditional control over responding to a CS by selectively activating memories of acquisition, rendering these memories, at the time of testing, more available than memories of extinction (see also Bouton \& Ricker, 1994). This account quite naturally explains the renewal phenome- 
non, a close cousin to reinstatement. This phenomenon refers to the increase in responding seen to an extinguished $\mathrm{CS}$ that is returned to the training context after it had been extinguished in an alternative context. By this account, the conditioning context acquires conditional control over acquisition memories, that is, the CS-US association, such that during the test the extinction memories are less effective at competing with acquisition memories to control performance.

Applying these ideas to the results, reported above, of Bouton and King (1986) requires an additional assumption. In these experiments, conditioning, extinction, and testing all occurred in the same context. In different groups, the reinstating USs were presented either in the same context as that used for testing or in a different context. Thus, it is not so plausible to assume, in this case, that the conditioning context can selectively retrieve acquisition over extinction memories. However, if it is assumed that the conditioned response to the context can acquire conditional control, then the results are quite sensibly explained. Bouton and King's experiments used a conditioned emotional response paradigm. If more fear is evoked by the context during conditioning than during extinction, then context-elicited conditioned fear could acquire conditional control over acquisition memories. Reinstating shocks presented in the test context following extinction would restore conditioned fear to the context and thereby enable it to cue acquisition memories selectively when the CS was subsequently tested there. The reinstatement phenomenon interpreted this way reduces to a special case of renewal.

Another prominent model of reinstatement also emphasizes conditional control mechanisms. However, instead of relying upon conditioned responses to the context to acquire conditional control over acquisition memories, the explanatory power rests in the signal properties acquired by the US itself. Several versions of this idea have been advanced (e.g., Bouton et al., 1993; Capaldi, 1994; Rescorla \& Skucy, 1969; Ricker \& Bouton, 1996), but the key assumption is that the outcome for one trial can serve to cue the subsequent trial. During acquisition, for instance, CS-US pairings enable the US from the previous trial to signal that the CS will be followed by the US on the subsequent trial. If the reinstating US is presented during the reinstatement test, responding to the CS should be elevated by this mechanism so long as the memory of the US is active at the time the CS is tested. Most studies of reinstatement have presented their reinstating USs $24 \mathrm{~h}$ prior to the reinstatement test. Capaldi's sequential theory (e.g., see Capaldi, 1994) has little difficulty with this fact, though, since it permits memories of the US to be retrieved by the context at the time that the CS test is to occur. With this assumption, the sequential model becomes rather similar to the view that context-elicited conditioned responses can acquire conditional control over responding. Note, however, that the sequential model, unlike the context occasion-setting model, is rather explicit in stating that the sequence of trials during the condi- tioning phase is critical in determining conditional control. The outcome of one trial should come to signal the trial that immediately follows it more than it should signal later trials.

Neither of the aforementioned theories of reinstatement were specifically designed to address the possibility of reinstatement that is reinforcer specific. The empirical search for outcome-specific reinstatement becomes a potential new testing ground for theories of reinstatement. The possibility of outcome-specific reinstatement in a rat appetitive conditioning paradigm is strengthened by at least a couple of findings. First, it is known that when rats learn to associate different CSs with different USs, they encode rather specific information about the outcomes (Colwill \& Motzkin, 1994; for a review, see Delamater \& LoLordo, 1991). Second, there is now good reason to believe that specific stimulus-outcome ( $\mathrm{S}-\mathrm{O}$ ) associations survive a variety of extinction treatments without any detectable impairment (Delamater, 1996; Rescorla, 1996). Given these results, it becomes of interest to ask whether different $\mathrm{S}-\mathrm{O}$ associations can be selectively reinstated.

The reinstatement literature contains isolated examples of outcome-general reinstatement (e.g., Baker et al., 1991; Shaham \& Stewart, 1995; Springer \& Miller, 1972), but, to my knowledge, no evidence suggesting the possibility of outcome-selective reinstatement has been documented. Thus, the empirical status of outcome-selective reinstatement is far from clear. In the first experiment reported here, this question was examined using a Pavlovian conditioning procedure; in the second experiment, evidence was sought for outcome-selective reinstatement using a discriminated instrumental conditioning procedure. Supportive evidence was observed in both experiments.

\section{EXPERIMENT 1}

Outcome-selective reinstatement was investigated using Pavlovian appetitive conditioning with a stimulus-elicited magazine approach as the index of learning (e.g., Bouton \& Peck, 1989; Farwell \& Ayres, 1979; Holland, 1979). Initially, two conditioned stimuli were reinforced with different outcomes during each acquisition session. Then, following a session of nonreinforced exposure to the stimuli, one of the reinforcers was presented during a single reinstatement test session in which both CSs were presented. Evidence for outcome-selective reinstatement would be obtained if different amounts of magazine-approach responses were evoked by the two stimuli in this test. It is important to note that several different trial sequences which guaranteed alternations of trial types to be more likely than repetitions were used throughout the acquisition phase. Under these conditions, the sequential theory would predict that each trial outcome would serve as a better discriminative cue for the other type of trial.

The context occasion-setting view predicts that reinstatement should occur equally to both CSs. If, during the conditioning phase, both types of CS-US trials occur in 
each session, the context should associate equally with each US. Context-elicited conditioned responses based upon these associations should therefore acquire conditional control over each type of CS-US trial. When the context's association with the reinstating US is subsequently strengthened after the extinction phase, this should permit the context to reinstate conditioned responding to each CS equally during the test. In other words, general, but not outcome-selective, reinstatement should be observed.

\section{Method}

\section{Subjects}

Sixteen male Sprague-Dawley rats, about 90 days old, were used. They were individually housed in a colony room on a 16:8-h light: dark cycle, and were maintained at $80 \%$ of their ad-lib body weights with daily supplemental feedings.

\begin{abstract}
Apparatus
The apparatus consisted of four identical conditioning chambers each of which was housed inside a sound- and light-resistant shell. The conditioning chambers measured $22.9 \times 20.3 \times 20.3 \mathrm{~cm}$. Two end walls were constructed of aluminum; the side walls and the ceiling were made of clear Plexiglas. The floor consisted of 0.48 $\mathrm{cm}$ stainless steel rods spaced $1.9 \mathrm{~cm}$ apart. Centered on one end wall $10 \mathrm{~mm}$ above the grid floor was a recessed magazine measuring $30 \times 36 \times 20 \mathrm{~mm}$ (length $\times$ width $\times$ depth). A single $45-\mathrm{mg}$ pellet (P. J. Noyes Co., Formula A) was dropped onto the magazine floor when this reinforcer was scheduled; $.3 \mathrm{ml}$ of an $8 \%$ sucrose solution was delivered through a gravity feed valve into a small well, the top of which was level with the magazine floor, when this reinforcer was scheduled. On the inner walls of the recessed magazine were an infrared detector and emitter used to record head movements inside the magazine. Located $2.5 \mathrm{~cm}$ to the left of the magazine and $8.9 \mathrm{~cm}$ above the floor was a standard lever manipulandum. Access to this lever was prevented in Experiment 1 by a sheet-metal covering. Mounted on the outer ceiling directly above the magazine was a $6-\mathrm{W}$ light bulb. During the visual CS (F), this light bulb flashed with a frequency of $1 / \mathrm{sec}$. Located above the chamber and attached to the back wall of the outer shell was a speaker through which a $1500-\mathrm{Hz}$ pure-tone stimulus $(\mathrm{T})$ was presented. This auditory stimulus measured $5 \mathrm{~dB}$ above a background level of $84 \mathrm{~dB}$ (C weighting). The chamber was always dark except when the visual conditioned stimulus was presented. Fans attached to the outer shells provided for cross-ventilation within the shell as well as background noise. All experimental events were controlled and recorded automatically by a microcomputer and interfacing equipment located in an adjacent room.
\end{abstract}

\section{Procedure}

Initially, rats were given one magazine training session with each reinforcer (first pellets and then sucrose). Each of these sessions was $20 \mathrm{~min}$ long, during which 20 reinforcers were delivered.

Over the next 10 days, all rats received conditioning sessions in which one of the stimuli was paired with pellets and the other paired with sucrose. Each stimulus was $10 \mathrm{sec}$ in duration, and the appropriate reinforcer was delivered at stimulus offset. The identity of the stimuli signaling sucrose and pellet was counterbalanced across animals. In every session, there were eight trials of each type, with an intertrial interval (ITI) averaging $2 \mathrm{~min}$ from stimulus onset to stimulus onset. The range of ITIs extended from 100 to $140 \mathrm{sec}$ Three different trial sequences were used, and these ensured that the probability of an alternation $(p=.79)$ of trial types was greater than the probability of a repetition $(p=.21)$ of trial types. Choice of these values ensured that each trial type was comparably distributed in time across the session. The different sequences consisted of the following trial orders of visual (V) and auditory (A) stimuli: VAAVAVVAVAAVVAVA (Sequence 1), AVVAVAAVAVVAAVAV (Sequence 2), and VAVAAVAVAVVAVAVA (Sequence 3). Magazine responses that occurred during the stimuli and during the 10sec interval just prior to the stimuli were recorded.

On the day following the 10 th conditioning session, the rats were given a single extinction session in which no reinforcers were presented. Otherwise, this extinction session was identical to the earlier acquisition sessions.

The reinstatement test occurred $24 \mathrm{~h}$ later during the next session. Prior to this test, the rats were individually assigned to one of two subgroups that were matched in terms of their responses to the stimuli on the preceding day's extinction session. As the reinstating event, one subgroup was given the test with pellets, and the other with sucrose. The session began with three presentations of the reinstating event, each separated by $30 \mathrm{sec}$. The first CS trial occurred $70 \mathrm{sec}$ after the third reinstating outcome. The first four CS trials alternated in a TFTF sequence. Note that the outcome associated with the tone CS was reinstated in this test for half of the subjects; the outcome associated with the flash CS was reinstated in this test for the remaining subjects. On average, the CSs were separated by a 2-min ITI, as in the earlier conditioning phase. The second half of the session was similar to the first half. Three additional presentations of the same reinstating event occurred, one every $30 \mathrm{sec}$. Then the stımuli were tested over four more trials. The order of these trials was FTFT. Magazine responses occurring in the 10-sec intervals prior to the CS, during the CS, and in each of two post-CS periods were recorded in this session.

\section{Statistical Analysis}

Analysis of variance (ANOVA) techniques were used to statistically evaluate the data. The conventional level of Type I error (.05) was used.

\section{Results and Discussion}

Acquisition of stimulus-evoked magazine responding over successive four-trial blocks of training is seen in Figure 1. This figure (as well as subsequent figures) portrays acquisition in terms of a difference score, where pretrial responses are subtracted from those occurring during the stimulus. In all of the analyses reported here, pretrial responding never differed between the two CSs. Furthermore, since the outcome-selective reinstatement effects to be reported did not interact with the identity of the reinstating event, pellets or sucrose, the data have been collapsed across that factor. The acquisition data in Figure 1 are presented separately for the CS whose outcome was subsequently reinstated (CSr) or not reinstated (CSnr). During each $C S$, acquisition of magazine responding reached a level that was considerably higher than that reached during the 10 -sec pretrial periods (approximately 27.0 responses/ $\mathrm{min}$ vs. 5.0 responses/min for each stimulus).

Figure 2 presents data from the extinction (left panel) and reinstatement (right panel) test sessions. The extinction data for each CS are presented in four-trial blocks, while the data for each CS from the four trials of the reinstatement test session have been combined. For the reinstatement test session, responding that occurred to each CS is shown, as is responding that occurred during each of two 10-sec post-CS intervals. In general, responding declined to each CS during the extinction session and more responding occurred during the reinstatement test to CSr than to CSnr. This effect was seen not only during 


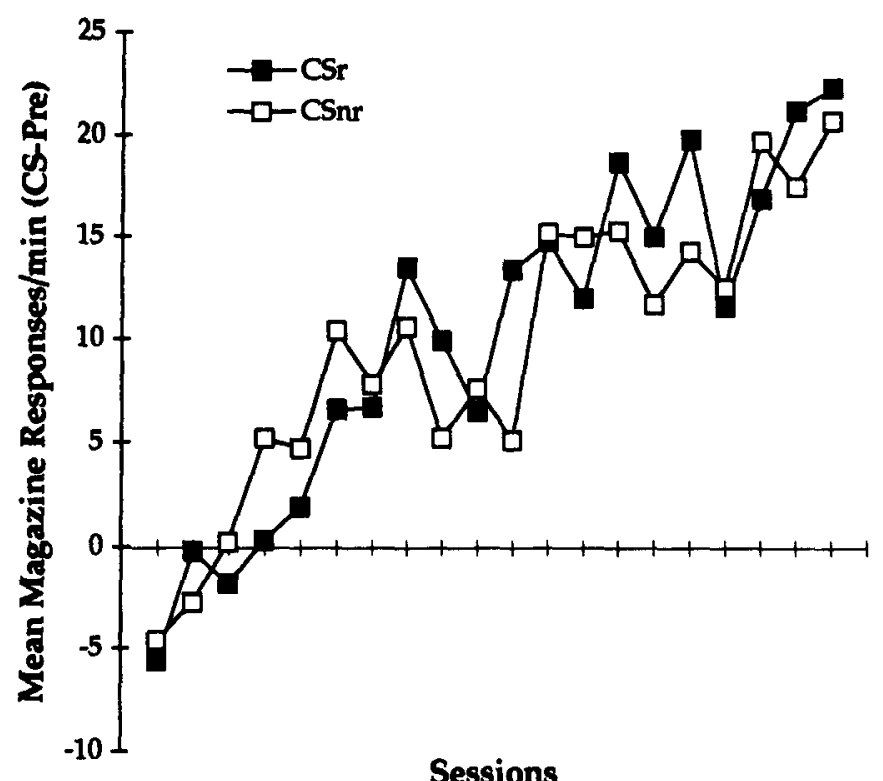

Figure 1. Mean magazine responding presented separately for the stimulus whose outcome would later be reinstated (CSr) or not (CSnr) over successive four-trial blocks of acquisition. There occurred two fourtrial blocks in each session.

the period in which the CS was present, but also during the two $10-$ sec post-CS intervals.

These observations were supported by statistical analyses. First, data from the final acquisition and the extinction sessions were analyzed in order to determine whether the single extinction session was successful at reducing conditioned responding. A session $\times \mathrm{CS} \times$ block ANOVA indicated that responding during the extinction session was reduced relative to the final acquisition session $[F(1,15)=$ 25.49], and that responding decreased over blocks during the extinction session but not during the final acquisition session $[F(1,15)=6.56]$. No other tests ieached statistical significance in this analysis.

A CS $\times$ interval ANOVA was performed on the reinstatement test data. This analysis revealed that responding during and after the $\mathrm{CS}$ whose outcome was reinstated was greater than responding during and after the CS whose outcome was not reinstated $[F(1,15)=6.81]$. This analysis also indicated that responding declined during the post-CS intervals relative to responding during the $\mathrm{CS}[F(2,30)=17.51]$. An analysis performed on the pre$\mathrm{CS}$ data indicated no difference between responding prior to $\mathrm{CSr}$ (3.6 responses $/ \mathrm{min}$ ) and $\mathrm{CSnr}$ ( 7.2 responses $/ \mathrm{min}$ ). The apparent difference was largely due to a single subject who responded at a level that was close to 4 standard deviations above the mean.

One final analysis was performed on these data. This analysis was designed to determine if the selective reinstatement treatment resulted in a selective increase in responding to the CS from the extinction session to the reinstatement test. Throughout the acquisition and extinction phases, responding to the two CSs were highly variable but quite frequently overlapping (see Figure 1). Although responding appears to have been somewhat greater to $\mathrm{CSr}$ than to CSnr in the final extinction block, the present analysis compared responding during the extinction session as a whole with responding during the reinstatement test session. This was thought to be reasonable since responding often appeared to be greater during CSnr than during CSr throughout acquisition and even during the first block of extinction. This analysis revealed a main effect neither of session nor of CS type, but the interaction between these two variables was marginally significant $[F(1,15)=4.28, p=.056]$.

To assess the stability of the selective reinstatement effect, a trial-by-trial analysis was performed on the CS data from the reinstatement test session (see Figure 3). As can be seen, the difference reported above occurred on every trial of the reinstatement test session. A CS $\times$ trial ANOVA indicated that responding was higher to CSr than to CSnr $[F(1,15)=4.71]$. Neither the trial nor the CS $\times$ trial interactions approached significance.

The present study demonstrates an outcome-selective reinstatement effect on stimulus-evoked magazine approach in a Pavlovian conditioning procedure. More magazine-approach responses occurred to the CS whose outcome had been reinstated during the test than to the CS whose outcome had not been presented during the test. Although responding to the two CSs clearly differed during the test, the data are somewhat ambiguous as to the degree of selectivity of outcome-selective reinstatement. Compared with the previous extinction session, it appeared as though responding did not increase to the CS whose outcome had not been reinstated. In other 


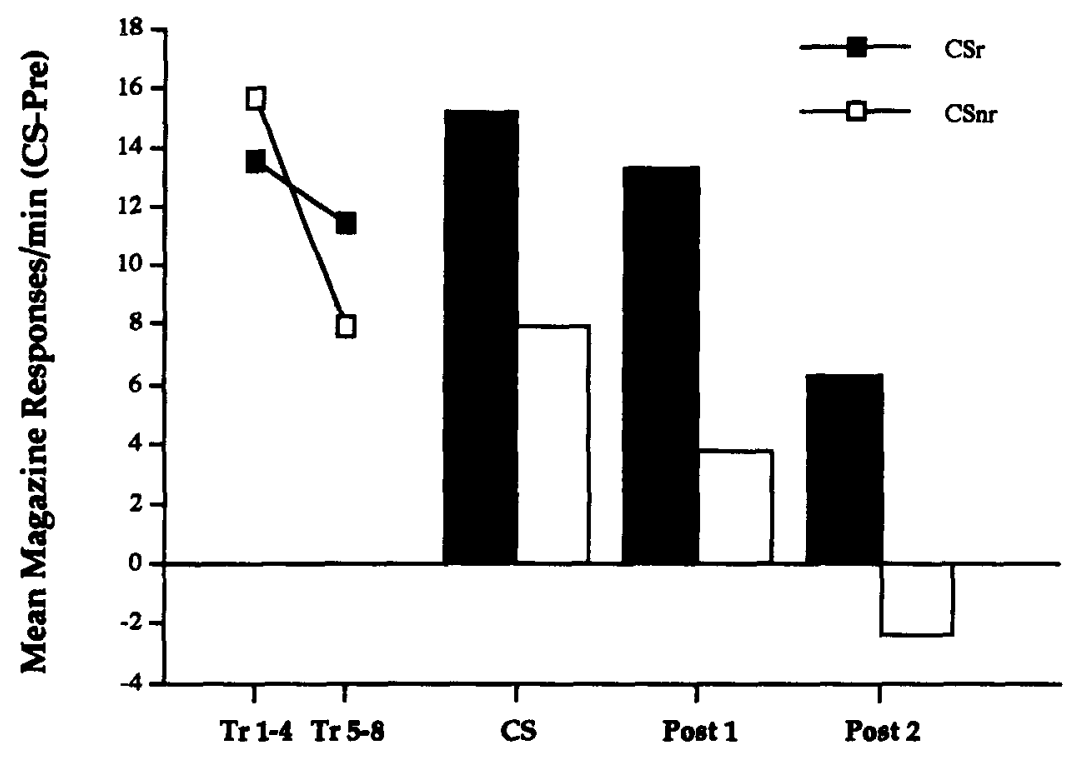

Extinction

Reinstatement

Figure 2. Mean magazine responding shown separately for the stimulus whose reinforcer was reinstated (CSr) or not reinstated (CSnr) during the extinction and reinstatement test sessions of Experiment 1 . For the test session, responding is shown during the CS as well as in each of two 10-sec post-CS intervals.

words, it appeared as though there was no evidence for general reinstatement. However, caution should be expressed on this point for at least a couple of reasons. First, there was no control group that failed to receive any reinstating USs. Without such a group, it is not known how subjects would have responded to the CSs without reinstating USs. Second, given that only one extinction session was administered in the present study, it is possible that the total amount of reinstatement was reduced due to the animals" being close to a "ceiling." A procedure that included more extensive extinction would be required to better assess the relative degrees of outcome-selective and outcome-general reinstatement effects. Such a procedure should be helpful for another reason as well. As noted above, the high degree of variability in the extinction data made it difficult to compare responding during the test session with responding during the extinction session. Only a marginally significant $\mathrm{CS} \times$ session effect was reported. The inclusion of more extinction sessions prior to the test should decrease the variability in the extinction data, permitting a better assessment of reinstatement. The second experiment was performed with this in mind.

\section{EXPERIMENT 2}

Experiment 1 demonstrated outcome-selective reinstatement of conditioned magazine responses using a Pavlovian conditioning procedure. The second experiment began to assess the generality of this effect by using an instrumental conditioning procedure. The learning of highly specific $\mathrm{S}-\mathrm{O}$ associations has been demonstrated in various discriminated instrumental conditioning pro- cedures (e.g., Colwill, 1994; Colwill \& Rescorla, 1988). Therefore, it seems likely that outcome-selective reinstatement might also occur in an instrumental discriminative conditioning procedure.

In Experiment 2, a discriminated operant procedure was used in which instrumental leverpressing was reinforced with one outcome in the presence of one discriminative stimulus, with a second outcome in the presence of a second discriminative stimulus, and nonreinforced during the ITI (A:R-O1, B:R-O2). Outcome-selective reinstatement was then assessed after extinction. Note that in this procedure, there is an opportunity to assess reinstatement of the discriminative control over magazine responding and instrumental leverpressing. The fact that responseoutcome associations have been shown to remain largely intact after a variety of extinction treatments (e.g., see Rescorla, 1991, 1992) suggests that outcome-selective reinstatement of this response might also be possible.

Another aim of the present experiment was to explore reinstatement after varying degrees of extinction in order to better assess the relative degrees of outcome-selective and outcome-general reinstatement. In Experiment 2, reinstatement was assessed both after a relatively brief (three sessions) and after a more extensive (eight additional sessions) extinction series. Finally, as in Experiment 1, the present experiment used a training procedure in which trials were more likely to alternate than to repeat.

\section{Method}

\section{Subjects and Apparatus}

A new set of 16 male Sprague-Dawley rats, approximately 90 days old, were used. They were maintained and run in the same 


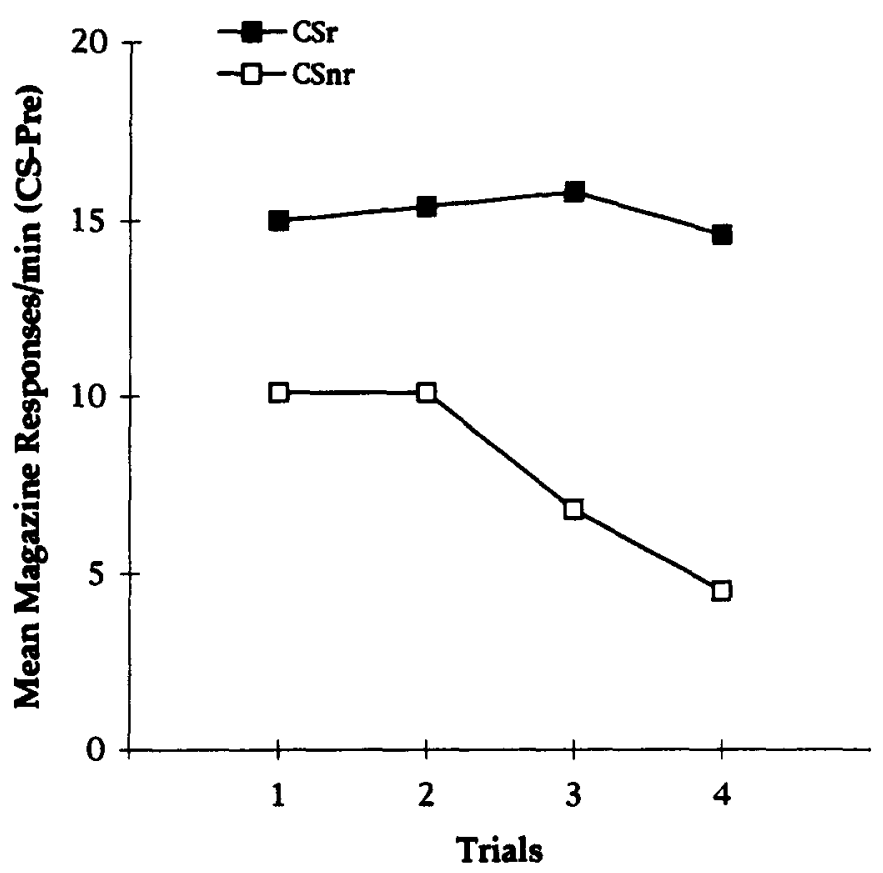

Figure 3. Mean magazine responding to CSr and CSnr during each trial of the reinstatement test session in Experiment 1.

apparatus as in the first experiment. In the present experiment, the sheet-metal cover preventing access to the lever manipulandum was removed.

\section{Procedure}

The rats were initially magazine trained as in Experıment 1. Following magazıne training, each rat was given instrumental cond1tioning with leverpresses continuously reinforced with one outcome until approximately 70 reinforcers were earned. Then, in a separate session, leverpresses were reinforced with the other outcome. The order in which pellets and sucrose were earned was counterbalanced across subjects.

Discriminative stimulus training. Discriminative stimulus training began on the following day and continued for 20 sessions. Each session contained eight 30 -sec trials in which the tone was presented and eight 30 -sec trials in which the flashing light was presented. During these stimulus periods, the leverpress response was reinforced according to a variable-interval 30 -sec schedule. One outcome could be earned in the presence of the tone and the other in the presence of the flashing light. The $\mathrm{S}-\mathrm{O}$ assignments were counterbalanced across animals. The ITI in the first session was $30 \mathrm{sec}$, measured from the offset of the stimulus to the next onset, and the trial sequence was VAAVAVVAVAAVVAVA. The ITI was increased to $60 \mathrm{sec}$ for each of the four subsequent sessions. Two trial sequences were used during these sessions, AVVAVAAVAVVAAVAV and VAVAAVAVAVVAVAAV. The ITI was increased to $90 \mathrm{sec}$ for the final 15 conditioning sessions. Two trial sequences were irregularly intermixed during these sessions, VAVAAVAVVAAVAVVA and AVAVVAVAAVVAVAAV. Note that use of these trial sequences produces a probability of alternation $(p=.74)$ from one trial type to the other, which is greater than the probability of a repetition ( $p=$ .26) of trial types.

Extinction and reinstatement testing. Reinstatement testing occurred after both brief and more extensive amounts of extinction experience. There was a total of three extınction sessions prior to the first set of reinstatement tests. An additional eight extinction sessions occurred before the second set of reinstatement tests. The extinction sessions were similar to the earlier training sessions, except that no reinforcers could be earned.

Each reinstatement test consisted of two sessions, one with each outcome serving as the reinstating event. The order in which the tests with each outcome occurred was counterbalanced. In the reinstatement test that followed the brief amount of extinction, these two sessions were separated by 2 days in which the animals remained in their home cages. In the reinstatement test that followed the extensive amount of extinction, these 2 sessions were separated by 2 additional days of extinction (to further discourage any residual effects of the first session from contaminating the second). The order of testing the two outcomes in the "extensive" test sessions was orthogonally related to the order of testing the two outcomes in the "brief" test sessions. For example, if an animal was tested with pellets and then sucrose as the reinstating outcomes in Tests 1 and 2 following brief extinction, half of the animals received this order in Tests 3 and 4 after extensive extinction and the remanning animals received the reverse order.

Reinstatement test sessions were structured in the following manner. The session began with a 90 -sec period in which three deliverles of the reinstating outcome occurred randomly in time. Two trials of each type then occurred in an ABBA sequence at 90-sec intervals thereafter (offset to onset). The second half of the session was run similarly, except that the trial sequence following the midsession $90-\mathrm{sec}$ reinstatement period was BAAB. Note that, as in Experiment 1 , three additional deliveries of the reinstating outcome occurred randomly in time during the midsession 90 -sec reinstatement period. The roles of $\mathrm{A}$ and $\mathrm{B}$ as discriminative stimuli whose outcome served as the reinstating event were counterbalanced across the animals.

\section{Results and Discussion}

The data from the discrimination training and extinction phases are shown in Figure 4. The top panel shows 


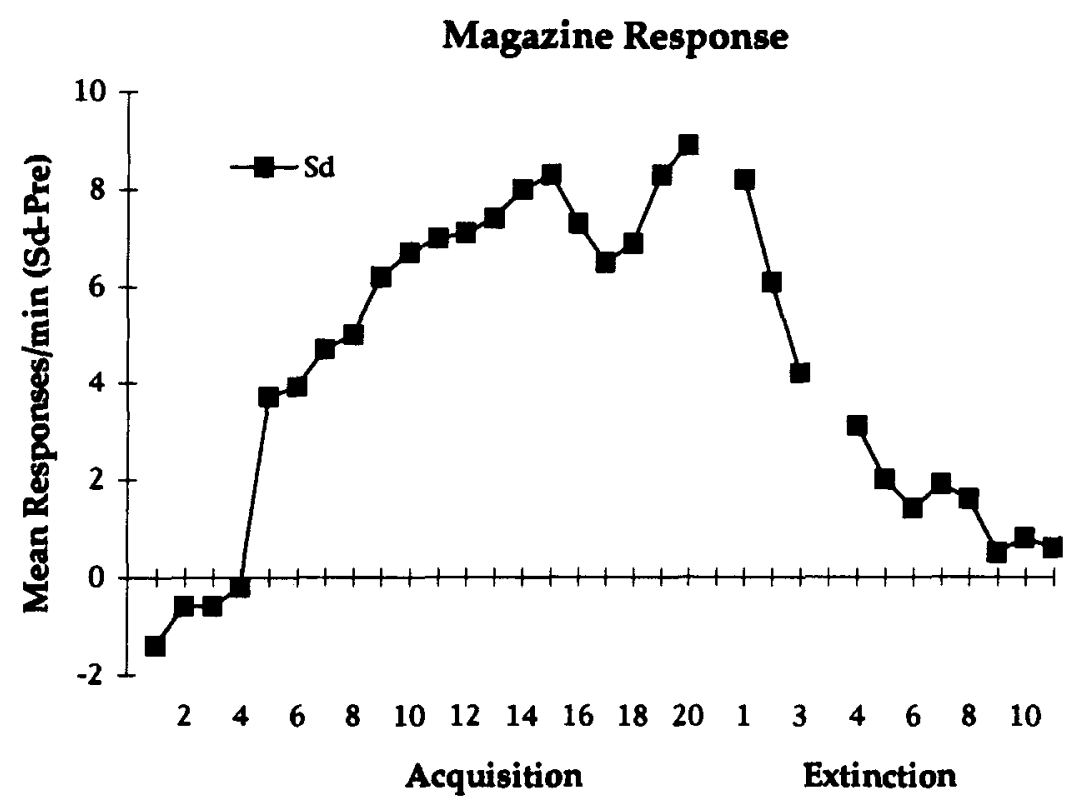

Leverpress Response

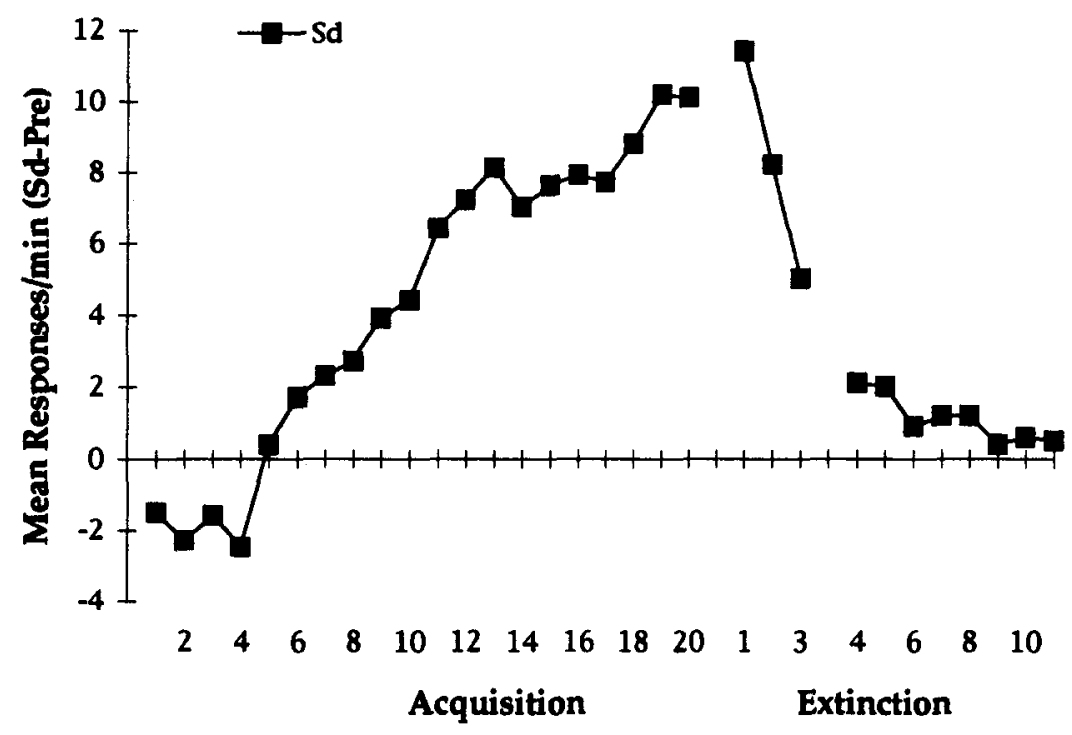

Figure 4. Mean magazine (upper panel) and leverpress responding (lower panel) occurring across the acquisition and extinction phases of Experiment 2 . Reinstatement tests occurred after brief and extensive extinction.

the magazine response data and the bottom panel shows the leverpress response data, collapsed across both types of discriminative stimulus trials. Once again, the data are expressed in terms of a difference score, which subtracted the rate of responding during the prestimulus interval from that occurring during the stimulus. On the final day of discrimination training, more magazine and leverpress responses occurred during the discriminative stimulus period (16.3 and 15.3 responses/min, respectively) than during the prestimulus periods ( 7.4 and 5.2 responses/ min, respectively). The figure also shows that extinction resulted in a rapid loss of discriminative magazine responding and leverpressing. This loss of discriminative performance reflected a decline in both prestimulus responding (not shown) and stimulus responding. The figure also shows that different levels of discriminative performance occurred after brief (first three sessions) and after more extensive (next eight sessions) amounts of extinction, with the latter reducing responding more completely. Compared with the final acquisition session, 
magazine responding $[F(1,15)=27.29]$ and leverpressing $[F(1,15)=61.42]$ were significantly reduced in the 3rd extinction session (decreasing to 5.9 and 5.5 responses/ min, respectively). Moreover, compared with the 3 rd extinction session, magazine responding $[F(1,15)=38.65]$ and leverpressing $[F(1,15)=48.46]$ were more completely reduced in the 11 th extinction session (decreasing to 1.2 and 0.6 responses $/ \mathrm{min}$, respectively).

The magazine response data from the reinstatement tests after brief (Tests 1 and 2) and extensive (Tests 3 and 4) amounts of extinction are presented in Figure 5. The data are broken down for each test session according to whether or not the outcome signaled by the discriminative stimulus was reinstated during that test session. For comparison, responding is also presented for each of the two discriminative stimuli during the final four-trial block of the extinction session which immediately preceded the test. Note that the discriminative stimulus whose outcome was reinstated in Test 1 (or 3 ) served as the stimulus whose outcome was not reinstated in Test 2 (or 4).

The data clearly indicate that presenting reinforcers during the test session generally increased responding to both discriminative stimuli over the levels seen in the prior extinction session. In addition, they are reasonably clear in showing that the reinstating effect of reinforcement in the test session was greater for the discriminative stimulus whose outcome was reinstated during the test. This selective reinstatement effect is clearly seen in each of the first three test sessions.
A four-way ANOVA performed on these data confirmed these impressions. The analysis included the factors of extinction (brief vs. extensive), the test session following extinction (first vs. second), the type of test session (extinction vs. reinstatement), and the type of discriminative stimulus (i.e., whether or not its outcome was reinstated during the test). The analysis revealed generally more responding in the tests that occurred after brief extinction than in those that occurred after extensive extinction $[F(1,15)=10.99]$. It also showed a general reinstatement effect as there was more responding during the test session than during the prior extinction session $[F(1,15)=29.13]$. Importantly, however, more responding was seen to the stimulus whose outcome was reınstated during the test, although this difference was not seen during the prior extinction session $[F(1,15)=6.36]$. No other main effects or interactions approached significance.

A separate four-way ANOVA was also performed on the prestimulus magazine-response data. The only significant effects revealed by this analysis were those showing more responding after brief than after extensive extinction $[F(1,15)=33.36]$, more responding during the reinstatement test sessions than during extinction $[F(1,15)=41.11]$, and an interaction between these two factors $[F(1,15)=7.21]$.

The corresponding leverpress data are presented in Figure 6 . The data clearly indicate that presenting reinforcers during the test session generally increased responding to the discriminative stimuli over the level seen in the

\section{Magazine Response}

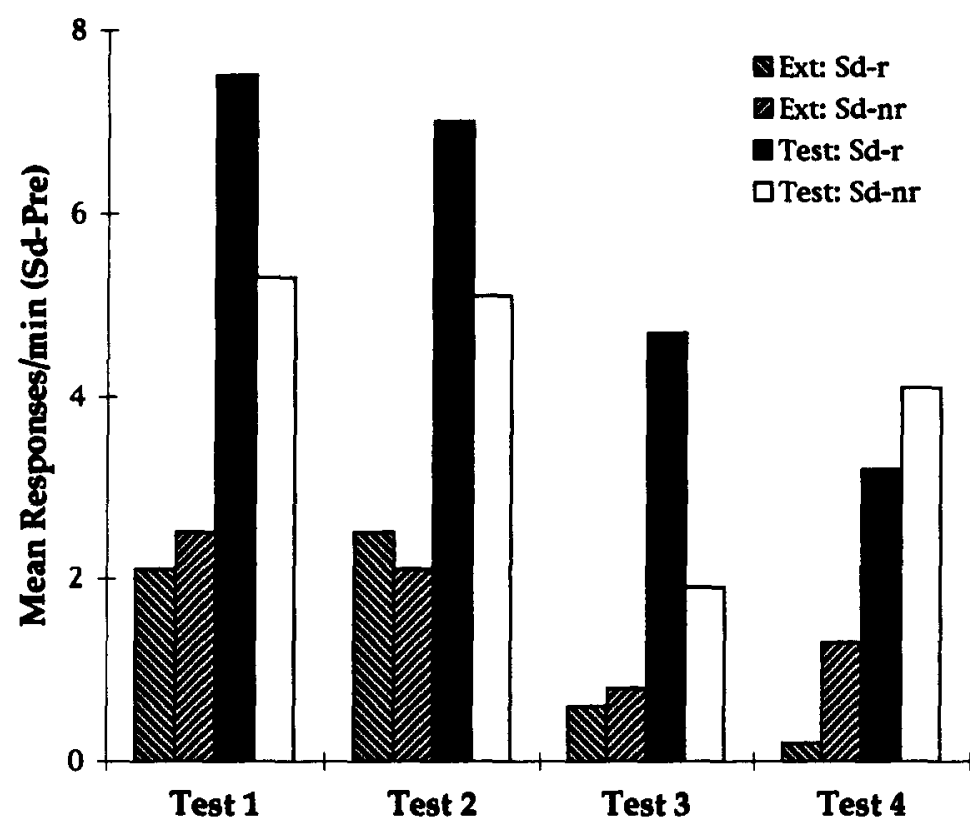

Figure 5. Mean magazine responding shown separately for the stimulus when its reinforcer was reinstated (Sd-r) or not reinstated (Sd-nr) during the reinstatement test sessions (Test) and during the immediately preceding extinction sessions (Ext) of Experiment 2. 


\section{Leverpress $\mathbf{F}$ nse}

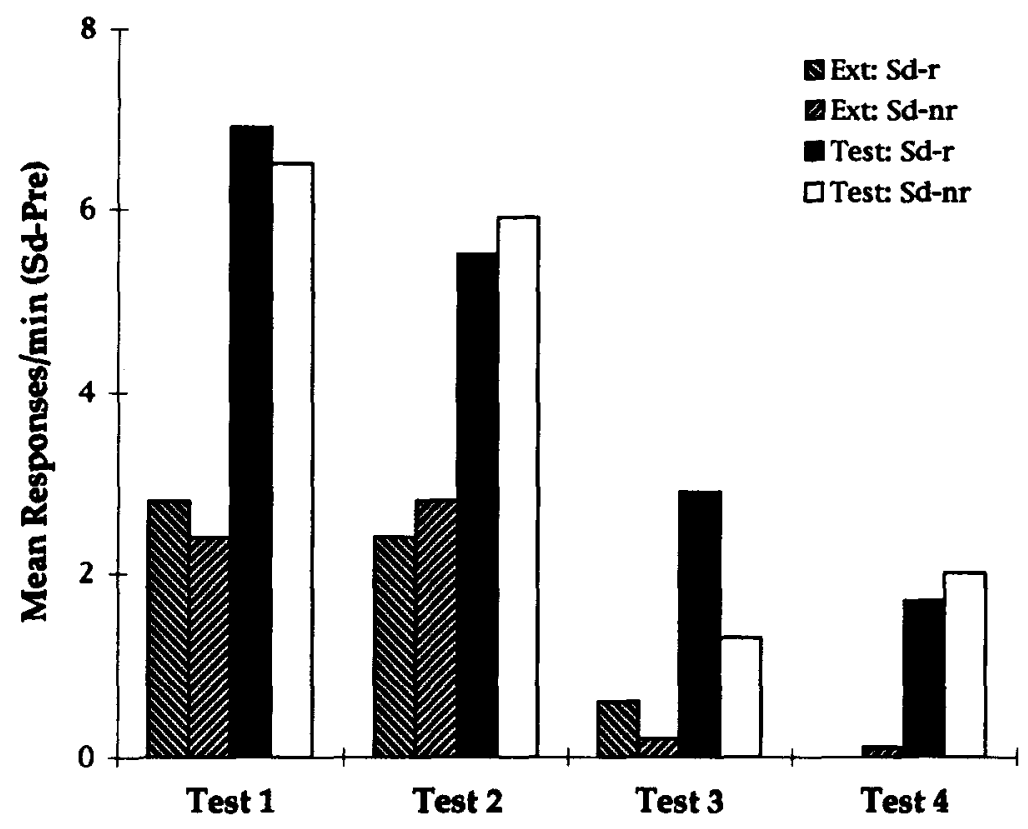

Figure 6. Mean leverpress responding shown separately for the stimulus when its reinforcer was reinstated (Sd-r) or not reinstated (Sd-nr) during the reinstatement test sessions (Test) and during the immediately preceding extinction sessions (Ext) of Experiment 2.

prior extinction session. However, they also show that selective reinstatement did not occur with this response.

A four-way ANOVA on the leverpress data revealed generally more responding in the tests that occurred after brief extinction than in those that occurred after extensive extinction $[F(1,15)=52.07]$. It also showed a general reinstatement effect, since there was more responding during the test sessions than during the prior extinction sessions $[F(1,15)=56.93]$. The only other significant effect was the interaction indicating a larger general reinstatement effect after brief than after extensive extinction $[F(1,15)=8.49]$. No other main effects or interactions approached significance.

A separate four-way ANOVA was also performed on the prestimulus leverpress response data. The only significant effects revealed by this analysis were those showing more responding after brief than after extensive extinction $[F(1,15)=11.12]$, more responding during the reinstatement test sessions than during extinction $[F(1,15)=21.73]$, and an interaction between these two factors $[F(1,15)=4.78]$.

An additional analysis was performed to more fully examine the time course of selective reinstatement effects. This analysis was motivated, in part, by the possibility that outcome-selective reinstatement of leverpressing might have occurred immediately after the reinstating outcome was presented. Recall that during the test sessions, reinstating outcomes were presented at the beginning and then in the middle of each test session. Two trials of each type occurred after each of these reinstatement periods.
It is possible that selective reinstatement effects are somewhat more transient than general reinstatement effects. Therefore, evidence of selective reinstatement might more easily be detected on the first test trial that occurred immediately after the reinstating event. To explore this possibility, a trials analysis was applied to the magazine and leverpress data. In particular, responding to the two discriminative stimuli was evaluated on each of the first two trials occurring after the reinstating outcome had been presented.

Magazine responding to the discriminative stimuli whose outcome was reinstated or not reinstated is presented in Figure 7 (upper panel) for the first and second trials occurring after the reinstatement periods during the brief and extensive extinction tests. In general, there appeared to be more responding in the presence of the stimulus whose outcome was reinstated than in the presence of the stimulus whose outcome was not reinstated. However, it also appeared as though more selective reinstatement occurred on the first trial than on the second trial after a brief amount of extinction, but that the reverse occurred after an extensive amount of extinction. Some of these impressions were statistically confirmed. A threeway ANOVA performed on these data, which included the factors of reinstatement condition, amount of extinction, and first versus second postreinstatement trial, revealed more overall responding in the presence of the stimulus whose outcome was reinstated than in the presence of the stimulus whose outcome was not reinstated $[F(1,15)=4.78]$. In addition, more overall responding 


\section{Magazine Response}

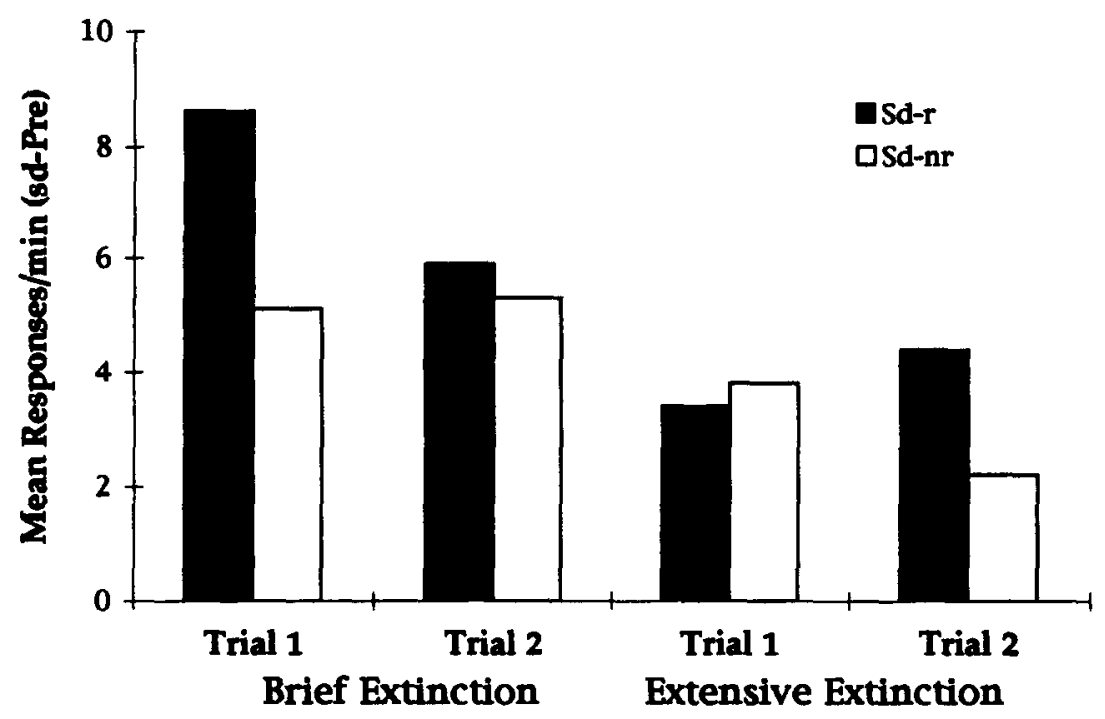

\section{Leverpress Response}

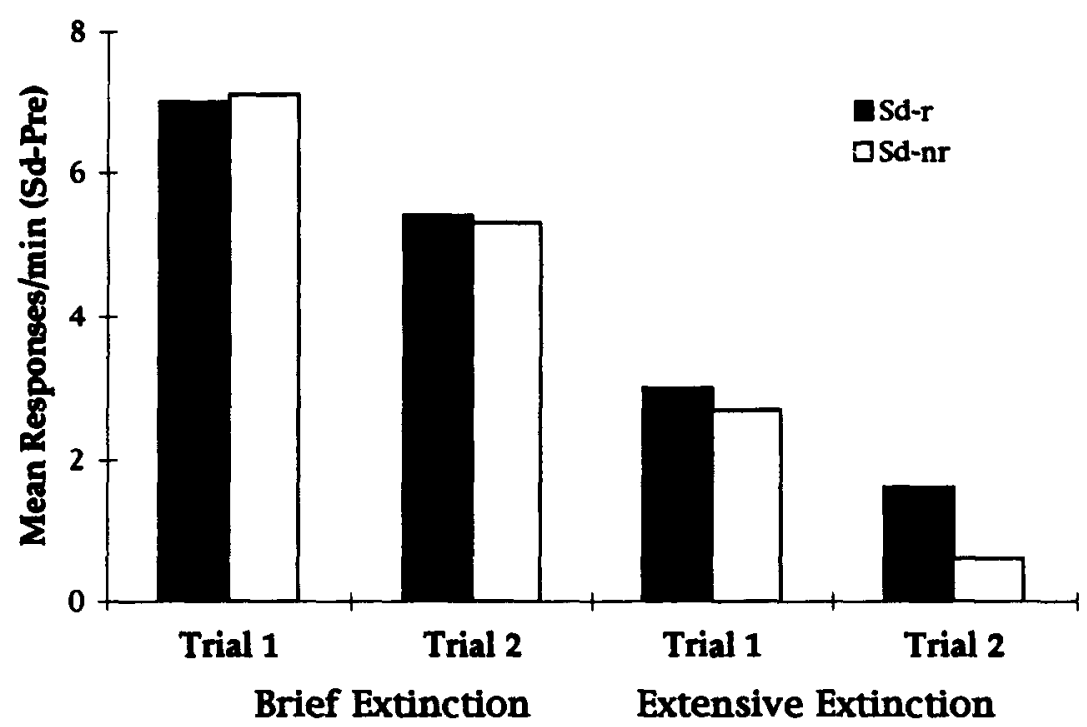

Figure 7. Mean magazine (upper panel) and leverpress responding (lower panel) shown separately for the stimulus whose outcome was reinstated (Sd-r) or not reinstated (Sd-nr) during each postreinstatement trial in the reinstatement test sessions following brief and extensive amounts of extinction.

occurred after brief than after extensive amounts of extinction $[F(1,15)=7.89]$. However, the apparent threeway interaction involving reinstatement condition, extinction condition, and trials fell short of conventional levels of significance $[F(1,15)=3.60, p=.08]$.

A similar analysis was performed on the leverpress data, shown in the lower panel of Figure 7. As was true for the session data presented in Figure 6, there was no strong evidence suggesting that leverpressing was selectively reinstated. It did appear, however, that there was more general reinstatement on the first trial than on the second trial following presentation of the reinstating outcomes, and generally more responding in the brief than in the extensive extinction tests. A three-way ANOVA performed on these data confirmed these impressions. The main effects of trials $[F(1,15)=10.88]$ and the amount of extinction $[F(1,15)=39.69]$ were both highly reliable. No other main effects or interactions approached statistical 
significance. Thus, even with this finer grain measure of reinstatement, there was no evidence for outcome-selective reinstatement of instrumental leverpressing.

In summary, the present data extend those from Experiment 1 in showing that outcome-selective reinstatement of conditioned magazine responses can be produced after discriminated instrumental training. Furthermore, selective reinstatement of conditioned magazine responding was observed after different levels of extinction were achieved. The magnitude of selective reinstatement seemed unaffected by that variable. Testing for reinstatement after more extensive extinction (than was used in Experiment 1) made it easier to estimate the degree to which reinstatement was selective. For example, if greater responding to the stimulus during the test session than during the prior extinction session can be taken as evidence for reinstatement, then the present experiment indicated both general and selective forms of reinstatement. In particular, the stimulus whose outcome was not reinstated during the test still elicited more magazine responding than it had in the prior extinction session. This conclusion should not be overstated, however, since there was no spontaneous recovery control group in the present study. Some of the nonspecific increase in responding could be attributed to that process.

It is also noteworthy that selective reinstatement of instrumental leverpressing was not observed, although a nonselective increase in leverpressing during the test was. One reason for this could have to do with competition introduced by an increased tendency to visit the magazine during the stimulus whose outcome was reinstated. This account, however, would require some statement about why increased magazine responding should compete with leverpressing and not vice versa. Another possibility is that introduction of noncontingent reinforcement during the test session might have selectively degraded the instrumental response-outcome contingency (Colwill \& Rescorla, 1986; Dickinson, Campos, Varga, \& Balleine, 1996; Williams, 1989). If this occurred, it should have decreased the tendency to leverpress during the stimulus whose outcome was reinstated. Although this possibility could account for the failure to observe selective reinstatement of leverpressing, it does not adequately explain the selective reinstatement of magazine responding given that noncontingent reinforcements should have selectively degraded the $\mathrm{S}-\mathrm{O}$ contingency as well (Delamater, 1995). Clearly, the difference in the sensitivity of magazine responding and leverpressing to selective reinstatement needs further analysis.

\section{GENERAL DISCUSSION}

The present data were consistent in demonstrating outcome-selective reinstatement of conditioned magazine responding, where more responses occurred to the stimulus whose outcome was presented as the reinstating event. In Experiment 1, such an effect was observed using a Pavlovian training procedure in which different outcomes were signaled by different stimuli in the same training sessions. In Experiment 2, that result was extended to a discriminated instrumental response procedure in which different outcomes could be earned by the same instrumental response in the presence of different discriminative stimuli in the same training sessions. However, although conditioned magazine responses were selectively reinstated in Experiment 2, instrumental leverpressing was not reinstated in an outcome-specific manner. Finally, evidence for general as well as selective reinstatement of magazine responding was obtained after varying amounts of extinction.

The present experiments were partly motivated by an empirical interest in the possibility of outcome-selective reinstatement, as well as by an interest in evaluating two currently popular theories of reinstatement. There are several interesting issues that arise from the data presented here. The first concerns the implications of outcomeselective reinstatement for theories of reinstatement. The second concerns the different sensitivities of magazine responding and leverpressing to outcome selective reinstatement. And the third issue concerns the implications of outcome-selective reinstatement for the detection of specific $\mathrm{S}-\mathrm{O}$ associations during Pavlovian and instrumental learning.

A noteworthy feature of the present experiments was that training consisted of the intermixing of two different trial types such that trials were more likely to alternate than they were to repeat. This procedural choice is important insofar as it may help to distinguish between the predictions of two currently popular theories of reinstatement. Bouton's (e.g., 1991) context occasion-setting theory of reinstatement holds that no outcome-selective reinstatement should have been obtained in the present experiments, since the context should associate equally with both outcomes during training. On the other hand, Capaldi's (e.g., 1994) sequential theory would predict that more responding should be observed to the stimulus whose outcome was not reinstated during the test. This prediction stems from the claim that the outcome for one trial can come to discriminatively cue the subsequent trial. Since trials were more likely to alternate than they were to repeat in the present studies, this suggests that each outcome should serve as a better discriminative cue for the other trial type.

In evaluating these ideas, a couple of important points should be considered. First, although the present experiments revealed evidence for outcome-selective reinstatement in a way not anticipated by sequential theory, no attempt was made to systematically examine the predictions of sequential theory. For instance, it seems possible that the training procedures used here might have underestimated the amount of outcome-selective reinstatement. Perhaps there would have been stronger outcome-selective reinstatement effects if trial alternation and repetition probabilities were equal or were balanced in favor of trial 
repetitions. This pattern of data would surely support the claims of the sequential theory. However, note that even if this were true, the sequential theory provides, at best, an incomplete explanation of the present results. Second, in support of the context occasion-setting view of reinstatement, it seems possible that the similarity between the occasion-setting stimulus (or "feature") and the outcome might play an important role in the acquisition of occasion setting. There exists evidence suggesting that stimulus similarity influences "simple" associative learning (e.g., Rescorla \& Furrow, 1977). It is not a far reach to suppose that similarity influences the course of occasion setting as well. In the present situations, all that needs to be assumed in order to retain the context occasion-setting view is that an outcome representation conditionally evoked by the context can more easily learn to occasionset same-outcome trials than different-outcome trials. The role of feature-outcome similarity in occasion setting, however, has yet to be determined.

Another approach concerns the possible relationship between reinstatement and spontaneous recovery. In the present studies, I have assumed that presentations of one outcome selectively increases conditioned responding to the stimulus associated with that outcome. Suppose, instead, that presentations of one outcome suppress spontaneous recovery of conditioned responding on the basis of the nonreinstated outcome. This possibility could accommodate the present results. It is more likely that this mechanism would apply to the results of Experiment 1 than to those of Experiment 2, however, since more extensive amounts of extinction were given in Experiment 2. Nevertheless, a spontaneous-recovery control group would be required to evaluate this claim. Such a possibility, though, still underscores the importance of outcomespecific processes in the present procedures, and this is the chief conclusion to be drawn here.

One additional approach to reinstatement should be mentioned. In some ways, the most straightforward account of the present results is based on the original notion of context-stimulus summation. To account for outcomeselective reinstatement, it would merely have to be assumed that greater summation occurs when the context and the stimulus have been associated with the same outcome than when they have been associated with different outcomes. Although this account is, perhaps, the most straightforward one, recall that this explanation was seriously questioned by Bouton and King's (1986; also Bouton et al., 1993) observations that context-stimulus summation does not always occur.

Quite apart from the implications of the present data for theories of reinstatement, another noteworthy feature of the present data was the different sensitivities shown by magazine responding and leverpressing in being selectively reinstated. On the assumption that conditioned magazine responses are Pavlovian conditioned responses (see also Balleine \& Dickinson, 1991), it follows that Pavlovian responses are more susceptible than instrumental responses to outcome-selective reinstatement. Al- though there have been demonstrations of reinstatement of instrumental responses (e.g., Baker et al., 1991; Rescorla \& Skucy, 1969), the present experiment is the first reported attempt to examine outcome-selective reinstatement of discriminated instrumental responses and Pavlovian conditioned responses concurrently. Such concurrent measurement might be especially powerful at highlighting this potential difference. Of course, it might be argued that magazine responses are really instrumental rather than Pavlovian in character. This argument cannot really be dismissed. However, as Rescorla (1994) has noted, these two types of responses do, at the very least, appear to differ quite a bit in their degree of instrumentality.

An alternative approach to the response-sensitivity issue may be provided by a more ethologically oriented framework. This approach would begin by noting that leverpressing and magazine behaviors differ not in terms of their Pavlovian or instrumental nature, but in terms of their relative proximity to food (see also Craig, 1918; Konorski, 1967; Timberlake \& Lucas, 1989). Perhaps reinstatement preferentially acts on those response "classes" which are more proximal to food. The notion that more specificity occurs with greater proximity to consummatory behaviors, such as eating or searching around a food hopper, is certainly not new (e.g., Konorski, 1967; Wagner \& Brandon, 1989). This framework suggests that magazine responding might be reinstated more selectively than leverpressing. However, a more thorough examination of this approach would require experimental control of proximal and distai behaviors in situations where outcome-selective reinstatement can be expected. Particularly interesting is the possibility that selective reinstatement of a normally distal behavior, such as instrumental leverpressing, would be observed in situations where it is made to be more proximal to food.

The final issue concerns the implications of the present data for studies that attempt to explore the status of specific $\mathrm{S}-\mathrm{O}$ associations after various extinction treatments. First, the very presence of an outcome-selective reinstatement effect demands that specific $\mathrm{S}-\mathrm{O}$ associations have been learned during training. Typically, the presence of specific $\mathrm{S}-\mathrm{O}$ associations have been measured using transfer of control (e.g., Delamater, 1996) or reinforcer devaluation techniques (e.g., Rescorla, 1996). The present studies can be viewed as developing an alternative technique for detecting the learning of specific $\mathrm{S}-\mathrm{O}$ associations. Used in this way, the present data suggest that specific $\mathrm{S}-\mathrm{O}$ associations have not only been learned but also have survived an extinction series. Thus, the data from the present situation may allow us to reach qualitatively similar conclusions to those reached using other measures of S-O associations (Delamater, 1996; Rescorla, 1996).

In conclusion, the present set of studies examined the possibility of outcome-selective reinstatement in situations in which currently popular models suggest that such reinstatement should not occur. While outcome-selective reinstatement of magazine responding was observed using 
both Pavlovian and instrumental conditioning procedures, instrumental leverpressing was shown to be reinstated only nonselectively. Although the present data can be interpreted in more than one way, they are consistent with additional research indicating that outcome-specific processes contribute importantly to conditioned performance.

\section{REFERENCES}

BaKer, A. G., Steinwald, H., \& Bouton, M. E. (1991). Contextual conditioning and reınstatement of extinguished instrumental responding. Quarterly Journal of Experimental Psychology, 43B, 199-218.

BALleINE, B., \& Dickinson, A. (1991). Instrumental performance following reinforcer devaluation depends upon incentive learning. Quarterly Journal of Experimental Psychology, 43B, 279-296.

Bouton, M. E. (1991). Context and retrieval in extinction and in other examples of interference in simple associative learning. In $\mathrm{L}$. Dachowski \& C. F. Flaherty (Eds.), Current topics in animal learning. Brain, emotion, and cognition (pp. 55-94). Hillsdale, NJ: Erlbaum.

Bouton, M. E., \& BolLEs, R. C. (1979). Role of conditioned contextual stimuli in reinstatement of extinguished fear. Journal of Experimental Psychology: Animal Behavior Processes, 5, 368-378.

Bouron, M. E., \& KING, D. A. (1986). Effect of context on performance to conditioned stimuli with mixed histories of reinforcement and nonreinforcement. Journal of Experimental Psychology: Animal Behavior Processes, 12, 4-15.

Bouton, M. E., \& PECK, C. A. (1989). Context effects on conditioning, extinction, and reinstatement in an appetitive conditioning preparation. Animal Learning \& Behavior, 17, 188-198.

Bouton, M. E., \& RickER, S. T. (1994). Renewal of extinguished responding in a second context. Animal Learning \& Behavior, 22 317-324.

Bouton, M. E., Rosengard, C., Achenbach, G. G., Peck, C. A., \& BrooKs, D. C. (1993). Effects of contextual conditioning and unconditional stimulus presentation on performance in appetitive conditioning. Quarterly Journal of Experimental Psychology, 46B, 63-95

CAPaLDI, E. J. (1994). The sequential view: From rapidly fading stimulus traces to the organization of memory and the abstract concept of number. Psychonomic Bulletin \& Review, 1, 156-181.

Colwill, R. M. (1994). Associative representations of instrumental contingencies. In D. L. Medin (Ed.), The psychology of learning and motivation (Vol. 28, pp. 1-72). New York: Academic Press.

ColwILl, R. M., \& MotZKIN, D. K. (1994). Encoding of the unconditioned stimulus in Pavlovian conditioning. Animal Learning \& Behavior, 22, 384-394.

Colwill, R. M., \& Rescorla, R. A. (1986). Associative structures in instrumental learning. In G. H. Bower (Ed.), The psychology of learning and motivation (Vol. 20, pp. 55-104). New York: Academic Press.

Colwil. , R. M., \& RESCORLA, R. A. (1988). Associations between the discriminative stimulus and the reinforcer in instrumental learning. Journal of Experimental Psychology: Animal Behavior Processes, $14,155-164$

Craig, W. (1918). Appetites and aversions as constituents of instincts. Biological Bulletin, 34, 91-107.

Delamater, A. R. (1995). Outcome-selective effects of intertrial reinforcement in a Pavlovian appetitive conditioning paradigm with rats. Animal Learning \& Behavior, 23, 31-39.

Delamater, A. R. (1996). Effects of several extinction treatments upon the integrity of Pavlovian stimulus-outcome associations. Animal Learning \& Behavior, 24, 437-449.

Delamater, A. R., \& LoLORDo, V. M. (1991). Event revaluation procedures and associative structures in Pavlovian conditioning. In L. Dachowski \& C. F. Flaherty (Eds.), Current topics in animal learning. Brain, emotion, and cognition (pp. 55-94). Hillsdale, NJ: Erlbaum.
Dickinson, A., Campos, J., Varga, Z. I., \& Balleine, B. (1996). Bidirectional instrumental conditioning. Quarterly Journal of Experimental Psychology, 49B, 289-306.

Farwell, B. J., \& AYRES, J. J. (1979). Stimulus reinforcer and response reinforcer relations in the control of conditioned appetitive headpoking (goal tracking) in rats. Learning \& Motivation, 10, 295-312.

HollaND, P. C. (1979). Differential effects of omission contingencies on various components of Pavlovian appetitive conditioned responding in rats. Journal of Experimental Psychology: Animal Behavior Processes, 5, 178-193.

KONORSKI, J. (1967). Integrative activity of the brain. Chicago: University of Chicago Press.

RESCORLA, R. A. (1991). Associations of multiple outcomes with an instrumental response. Journal of Experimental Psychology: Animal Behavior Processes, 17, 465-474.

Rescorla, R. A. (1992). Associations between an instrumental discriminative stimulus and multiple outcomes. Journal of Experimental Psychology: Animal Behavior Processes, 18, 95-104.

RESCORLA, R. A. (1994). Control of instrumental performance by Pavlovian and instrumental stimuli. Journal of Experimental Psychology: Animal Behavior Processes, 20, 44-50.

RESCORLA, R. A. (1996). Preservation of Pavlovian associations through extinction. Quarterly Journal of Experimental Psychology, 49B, 245-258.

RESCORLA, R. A., \& FurRow, D. R. (1977). Stimulus similarity as a determinant of Pavlovian conditioning. Journal of Experimental Psychology: Animal Behavior Processes, 3, 203-215.

RESCORLA, R. A., \& HeTH, C. D. (1975). Reinstatement of fear to an extinguished conditioned stimulus. Journal of Experimental Psychology: Animal Behavior Processes, 1, 88-96.

RESCORLA, R. A., \& SKUCY, J. C. (1969). Effect of response-independent reinforcers during extinction. Journal of Comparative \& Physiological Psychology, 67, 381-389.

RICKeR, S. T., \& BouTON, M. E. (1996). Reacquisition following extinction in appetitive conditioning. Animal Learning \& Behavior, 24, 423-436.

Schachtman, T. R., Brown, A. M., \& Miller, R. R. (1985). Reinstatement-induced recovery of a taste- $\mathrm{LiCl}$ association following extinction. Animal Learning \& Behavior, 13, 223-227.

ShaHAM, Y., Rodaros, D., \& SteWART, J. (1994). Reinstatement of heroin-reinforced behavior following long-term extinction: Implications for the treatment of relapse to drug taking. Behavioural Pharmacology, 5, 360-364.

SHAHAM, Y., \& STEWART, J. (1995). Stress reinstates heroin-seeking in drug-free animals: An effect mimicking heroin, not withdrawal. Psychopharmacology, 119, 334-341.

SPRINGER, A. D., \& MiLleR, R. R. (1972). Retrieval failure induced by electroconvulsive shock: Reversal with dissimilar training and recovery agents. Science, 177, 628-630.

Timberlake, W., \& Lucas, G. A. (1989). Behavior systems and learning: From misbehavior to general principles. In S. B. Klein \& R. R. Mowrer (Eds.), Contemporary learning theories: Instrumental conditioning theory and the impact of biological constraints on learning (pp. 237-275). Hillsdale, NJ: Erlbaum.

WAGNER, A. R., \& Brandon, S. E. (1989). Evolution of a structured connectionist model of Pavlovian conditioning (AESOP). In S. B. Klein \& R. R. Mowrer (Eds.), Contemporary learning theories: Instrumental conditioning theory and the impact of biological constraints on learning (pp. 149-189). Hillsdale, NJ: Erlbaum.

WILLIAMS, B. A. (1989). The effects of response contingency and reinforcement identity on response suppression by alternative reinforcement. Learning \& Motivation, 20, 204-224.

(Manuscript received March 17, 1997; revision accepted for publication June 4, 1997.) 